\title{
Comportement dynamique non linéaire d'un arbre vertical de turbopompe monté dans des paliers lubrifiés à l'eau
}

\author{
Non linear dynamic behavior of a vertical canned motor pump shaft \\ mounted on water lubricated journal bearings
}

par O. Bonneau, J. Frêne

Laboratoire de Mécanique des Solides — UMR CNRS 6610, Université de Poitiers

\author{
J.L. Killian
}

Technicatome

The dynamic behavior prediction of vertical canned motor pump shaft is very important for industrial applications. The performances and reliability of these components depend on a large part of the dynamic behavior. The present study characterizes the behavior of a vertical shaft mounted on two water lubricated journal bearings under radial magnetic and impeller load. Trajectories in the bearings are very unstable and the modelisation is presented and the obtained results on a vertical shaft are commented.

The rotor calculation is a step by step non linear computation with at each step the resolution of the pressure field in journal bearings. These pressure fields are obtained by resolution (finite differences) of the differential Reynolds Equation. The shaft is taken rigid with 4 degrees of freedom.

This study confirms the high level of unstability of a vertical shaft mounted in journal bearings. The main reason of that unstability is the pressure feed $(0.45 \mathrm{MPa}$ and $0.8 \mathrm{MPa})$, in association with magnetic attraction and with a low radial load.

\section{IINTRODUCTION}

Les paliers hydrodynamiques ont un comportement dynamique qui peut être très complexe. Pour de nombreuses applications, une linéarisation de ce comportement peut être effectuée sous certaines hypothèses mais il est bien connu que le caractère très instable des arbres verticaux montés sur paliers conduit à un fort comportement non linéaire de ceuxci.

Plusieurs travaux ont permis de modéliser le comportement des paliers fluides. Dès 1924 , Newkirk $[1,2]$ montre expérimentalement l'apparition d'une fréquence de vibration due à la présence du fluide dans le palier. Par la suite Hummel [3] et de nouveau Newkirk [4] effectuèrent un travail expérimental confirmant l'instabilité due au palier fluide. Ils notè- rent que pour des petites valeurs d'excentricité le rotor était animé d'un mouvement de précession avec une fréquence proche de la demi-fréquence de rotation (half frequency whirl), cette précession disparaissant lorsque l'excentricité augmentait. En 1956, Hori [5] détermina les 8 coefficients dynamiques de la théorie linéaire développée par Stodola [6] ; il utilisa pour cela les hypothèses du palier infiniment long, ce qui lui permit de montrer analytiquement la stabilité ou l'instabilité de différentes configurations d'arbres rigides montés sur paliers fluides. Par la suite, Holmes [7] confirma, en utilisant la théorie du palier court, les résultats obtenus par Hori.

La modélisation complète des paliers (paliers de longueur finie) fut développée quelques années plus tard grâce à l'arrivée des outils de calcul modernes (Sternlicht [8] and Lund [9]). 
Holmes [10, 11], Lund [11] et Abdul Wahed [13] développèrent des outils de simulation complets permettant de prédire le comportement non linéaire des rotors montés sur paliers fluides et soumis à de forts balourds.

L'étude présentée ici concerne une simulation du comportement dynamique non linéaire d'un arbre vertical de turbopompe monté dans deux paliers fluides lubrifiés à l'eau. Les différentes modélisations adoptées seront présentées ainsi que des résultats correspondant à une application industrielle.

\section{II $\square$ MODÉLISATION}

\subsection{Palier fluide}

\subsubsection{Champ de pression}

La recherche des actions mécaniques générées par le fluide sur l'arbre nécessite la recherche du champ de pression au sein du film.

Les hypothèses générales de la mécanique des films minces permettent de simplifier les équations de la mécanique des milieux continus et d'obtenir, dans le cas d'un palier en écoulement laminaire, une équation aux dérivées partielles du second ordre en termes de pression :

$$
\frac{1}{R^{2} \partial \theta}\left[\frac{h^{3} \partial P}{\mu k_{x} \partial \theta}\right]+\frac{\partial}{\partial z}\left[\frac{h^{3} \partial P}{\mu k_{z} \partial z}\right]=\frac{\omega \partial h}{2 \partial \theta}+\dot{x} \cos \theta+\dot{y} \sin \theta
$$

\section{Equation de Reynolds}

Les divers paramètres (voir aussi fig. 1) intervenant dans l'équation sont les suivants :

$-R$ : rayon du palier.

$-\omega$ : vitesse de rotation.

$-\mu$ : viscosité de l'huile.

$-\theta$ : coordonnée angulaire dont l'origine est située sur la ligne des centres.

$-z$ : coordonnée longitudinale.

- $h$ : épaisseur du film d'huile $=C(1+\varepsilon \cos \theta)$.

- $(\dot{x}, \dot{y})$ : vitesse du centre de l'arbre par rapport au coussinet. $-\varepsilon$ : excentricité relative.

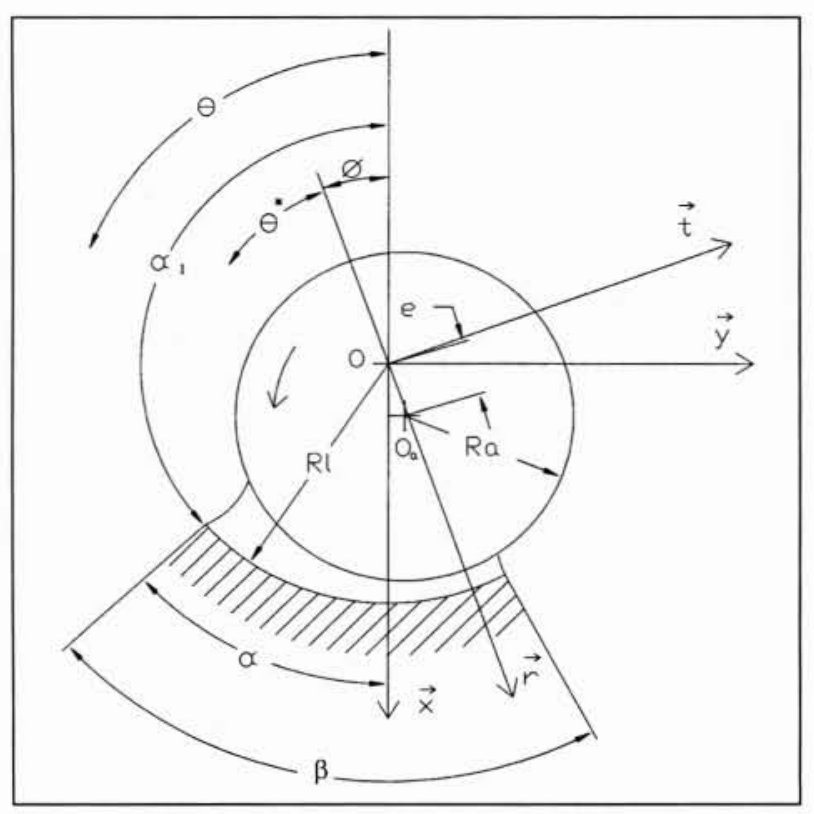

1. Caractéristique du film.
Dans le cas de fluides peu visqueux, de jeux de fonctionnement importants, de vitesses de rotation et gradients de pression élevés, l'écoulement n'est plus laminaire et deux coefficients de turbulence, $k_{x}$ et $k_{z}$ sont introduits ; ils permettent de tenir compte des effets dus à la turbulence. Ces coefficients dépendent des paramètres de l'écoulement : nombre de Reynolds local $\left(R_{e}\right)$ et gradients de pression en $z$ et en $\theta$. Ils sont calculés pour toute une plage de valeurs de ces trois paramètres. Une tabulation de $k_{x}$ et $k_{z}$ peut être ensuite utilisée pour effectuer un calcul de champ de pression. Différents modèles de turbulence ont été appliqués [14, $15,16,17]$ afin de calculer ces deux coefficients. En raison des faibles nombres de Reynolds apparaissant dans l'étude présentée ici, une formulation linéarisée développée par Constantinescu [18] est utilisée :

$$
\begin{aligned}
& k_{x}=12+0,0136 R_{e}^{0,9} \\
& k_{z}=12+0,0043 R_{e}^{0.96}
\end{aligned}
$$

Différentes conditions aux limites doivent être appliquées afin de résoudre l'équation de Reynolds :

- des conditions sur l'alimentation du palier (rainures, trous d'alimentation),

- des conditions sur la rupture du film :

- conditions de Sommerfeld (film complet),

- conditions de Gumbel (film d'amplitude $\pi$ ),

- condition de Reynolds.

L'équation de Reynolds est alors discrétisée par une méthode du type "différences finies" et l'intégration du champ de pression permet d'obtenir les forces exercées par le film fluide sur l'arbre :

$$
\begin{aligned}
& F_{x}=\int_{1 / 2}^{1 / 2} \int_{\theta_{2}}^{\theta_{1}} P(\theta, z) \cos \theta R d \theta d z \\
& F_{y}=\int_{1 / 2}^{1 / 2} \int_{\theta_{2}}^{\theta_{1}} P(\theta, z) \cos \theta R d \theta d z
\end{aligned}
$$

\subsubsection{Paliers mésalignés}

La prise en compte du mésalignement du palier introduit un couple de rappel dû à la dissymétrie axiale du champ de pression.

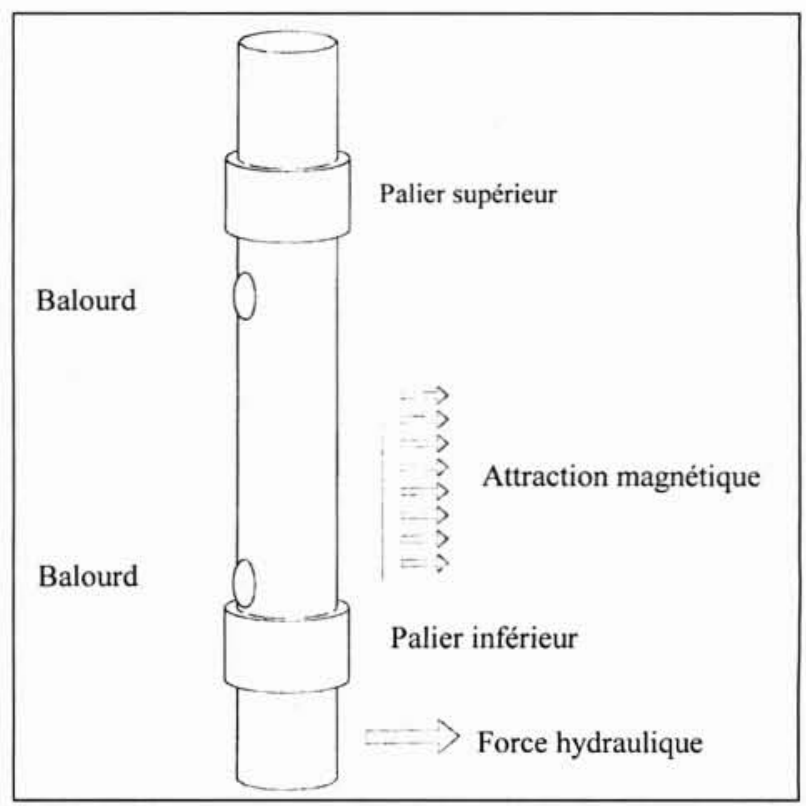

2. Charges sur le rotor. 
Le mésalignement du rotor est caractérisé par les deux angles d'Euler notés ici $\alpha$ et $\beta$, l'épaisseur du film fluide prend alors la forme suivante :

$$
h(\theta, z)=(x+z \beta) \cos \theta+(y-z \alpha) \sin \theta+C
$$

Le mésalignement dynamique modifie en partie le deuxième membre de l'équation de Reynolds qui devient :

$$
\frac{\omega \partial}{2} \partial \theta+(\dot{x}+\dot{\beta} z) \cos \theta+(\dot{y}-\dot{\alpha} z) \sin \theta
$$

\subsection{Modélisation du rotor}

Dans l'étude présentée ici le rotor est supposé rigide (en effet ses vitesses de rotation sont très petites comparées à sa première fréquence critique).

Quatre paramètres suffisent donc pour caractériser sa position spatiale : $x$ et $y$, représentant la position du centre de gravité de l'arbre et les deux angles d'Euler $\alpha$ et $\beta$.

Les équations de Lagrange appliquées à l'arbre permettent d'écrire les équations du mouvement :

$$
\frac{d \partial E_{c}}{d t \partial \dot{q}_{i}}-\frac{\partial E_{c}}{\partial q_{i}}=\left\{\begin{array}{c}
m \ddot{x} \\
\ddot{m} \\
A \ddot{\alpha}+\omega \dot{\beta} I \\
A \ddot{\beta} \omega \dot{\alpha} I
\end{array}\right\}=\Sigma \text { Torseur des forces }
$$

avec $A$ le moment d'inertie de l'arbre par rapport à un diamètre et $I$ le moment d'inertie par rapport à l'axe de rotation ( $I \omega \alpha$ et $I \omega \beta$ caractérisent les effets gyroscopiques).

\subsection{Modélisation des autres interactions}

La figure 2 présente la position des différentes forces exercées sur le rotor.

\subsubsection{Attraction magnétique}

Le rotor évoluant dans un champ magnétique, il apparaît une force d'attraction magnétique.

Cette attraction s'exerce entre les abscisses : $l_{0}=-0,131 \mathrm{~m}$ et $l_{1}=-0,051 \mathrm{~m}$ avec une force sur un élément de longueur du type : $F_{\text {Mag }}=k^{*} e^{*} \Delta l$ avec $k=12,0810^{6}, e$ l'excentrement de la tranche considérée (en $\mathrm{m}$ ), $\Delta l$ la longueur de la tranche considérée (en $\mathrm{m}$ ) et la charge exprimée en $N$; cette charge est une charge du type attraction avec une direction locale orientée selon la ligne des centres.

Cet élément de force est intégré analytiquement et le torseur correspondant est exprimé au centre de gravité de l'arbre :

$$
\left\{\begin{array}{l}
F_{x}=+k x_{G}\left(l_{1}-l_{0}\right)+k \frac{\beta}{2}\left(l_{1}^{2}-l_{0}^{2}\right) \\
F_{y}=+k y_{G}\left(l_{1}-l_{0}\right)-k \frac{\alpha}{2}\left(l_{1}^{2}-l_{0}^{2}\right) \\
M_{x}=-\frac{k}{2} y_{G}\left(l_{1}^{2}-l_{0}^{2}\right)+k \frac{\alpha}{3}\left(l_{1}^{3}-l_{0}^{3}\right) \\
M_{y}=+\frac{k}{2} x_{G}\left(l_{1}^{2}-l_{0}^{2}\right)+k \frac{\beta}{3}\left(l_{1}^{3}-l_{0}^{3}\right)
\end{array}\right.
$$

avec $x_{\text {e }}$ et $y_{\text {g }}$ les positions dú centre de gravité.

\subsubsection{Confinement du fluide}

Une force hydrodynamique est créée par la présence d'une zone de liquide confiné. La charge locale est choisie de la forme suivante :

$$
F_{\text {conf }}=\rho(\omega-2 \Phi)^{2} * \frac{R^{3} * \pi(1-\varepsilon)^{2} \varepsilon}{4\left(1-\varepsilon^{2}\right)^{\frac{3}{2}}} * \Delta l
$$

Elle s'exerce entre les abscisses $-0,161 \mathrm{~m}$ et $-0,021 \mathrm{~m}$, $R=0,0586-5 \mathrm{~m} \quad$ et $\varepsilon=e / C$ avec $C=1,1 \cdot 10^{-3} \mathrm{~m}$, $\rho=971,8 \mathrm{~kg} / \mathrm{m}^{3}$.

Cette charge locale est intégrée numériquement, mais elle a une valeur qui est négligeable vis-à-vis des autres charges en présence.

\subsubsection{Les balourds}

Nous allons considérer dans cette étude deux familles de balourds :

- deux balourds magnétiques de $6 \mathrm{~g} . \mathrm{cm}$ situés dans les plans d'abscisse $-0,157 \mathrm{~m}$ et $0,003 \mathrm{~m}$,

- un balourd hydraulique constitué d'une poussée hydraulique radiale fixe de $50 \mathrm{~N}$ et d'une poussée hydraulique fluctuante synchrone de $100 \mathrm{~N}$.

\subsection{Calcul non linéaire}

La simulation du comportement dynamique non linéaire de l'arbre vertical monté sur paliers fluides est une résolution pas à pas du principe fondamental de la dynamique appliqué au rotor et de l'équation de Reynolds. Le déroulement du calcul est le suivant :

1. initialisation des vitesses et positions de l'arbre,

2. calcul de toutes les forces extérieures,

3. calcul des forces non linéaires dans les paliers (résolution de l'équation de Reynolds),

4. calcul des accélérations de l'arbre,

5. intégration des accélérations (méthode d'Euler à pas variable),

6 . le calcul reprend alors en 2 .

\section{III — RÉSULTATS}

\subsection{Données}

Le calcul non linéaire de l'arbre vertical monté sur deux paliers fluides est effectuée pendant $0,6 \mathrm{~s}$, ce qui correspond à 36 tours de l'arbre. Ce temps est suffisamment long pour éliminer toute la partie transitoire (transitoire mécanique et numérique). Les tableaux 1 et 2 présentent les principales données du problème traité.

Tableau 1. - Données de l'arbre.

\begin{tabular}{|l|c|}
\hline Masse & $15 \mathrm{~kg}$ \\
Inertie $(A)$ en $G$, axe $\perp$ à axe de rotation & $0,4 \mathrm{~kg} \cdot \mathrm{m}^{2}$ \\
Inertie (/) par rapport à I'axe de rotation & $0,07 \mathrm{~kg} \cdot \mathrm{m}^{2}$ \\
Position du palier supérieur $/ G$ & $0,232 \mathrm{~m}$ \\
Position du palier inférieur $/ G$ & $0,145 \mathrm{~m}$ \\
\hline
\end{tabular}

\section{- 3.2 Charges dans les paliers}

La figure 3 présente les trajectoires de charges exercées par les deux paliers sur l'arbre.

Les figures 4 et 5 présentent les composantes en $x$ et $y$ de ces charges en fonction du temps. Le calcul non linéaire a été effectué pendant $0,6 \mathrm{~s}$ mais seules les 0,1 dernières secondes sont représentées, ce qui correspond à 6 tours de l'arbre. 
Tableau 2. - Données des paliers.

\begin{tabular}{|l|l|l|}
\hline & $\begin{array}{l}\text { Palier } \\
\text { supérieur }\end{array}$ & $\begin{array}{l}\text { Palier } \\
\text { inférieur }\end{array}$ \\
\hline Diamètre & $40 \mathrm{~mm}$ & $40 \mathrm{~mm}$ \\
Longueur & $60 \mathrm{~mm}$ & $70 \mathrm{~mm}$ \\
Jeu radial & $0,053 \mathrm{~mm}$ & $0,053 \mathrm{~mm}$ \\
Vitesse de rotation & $3600 \mathrm{tr} / \mathrm{mn}$ & $3600 \mathrm{tr} / \mathrm{mn}$ \\
Pression d'alimentation & 4,5 bars & 8 bars \\
Tension de vapeur saturante & $0,85 \mathrm{bars}$ & $0,48 \mathrm{bars}$ \\
Viscosité cinématique & $29510^{-6} \mathrm{Pa.s}$ & $35110^{-6}$ \\
Masse volumique & $961,9 \mathrm{~kg} / \mathrm{m}^{3}$ & $971,8 \mathrm{~kg} / \mathrm{m}^{3}$ \\
Température & $95^{\circ} \mathrm{C}$ & $80^{\circ} \mathrm{C}$ \\
\hline
\end{tabular}

Le palier inférieur est très influencé par le balourd hydraulique, ce qui est logique en raison de la position du point d'application de ce balourd. Ce balourd s'exerce à la fréquence de rotation de l'arbre. Sur cette première charge se superpose l'attraction magnétique qui, elle, agit avec une fréquence égale à la fréquence de précession de l'arbre (demi-fréquence). Cette superposition se traduit par l'apparition de deux boucles (fig. 3 et 4 ).

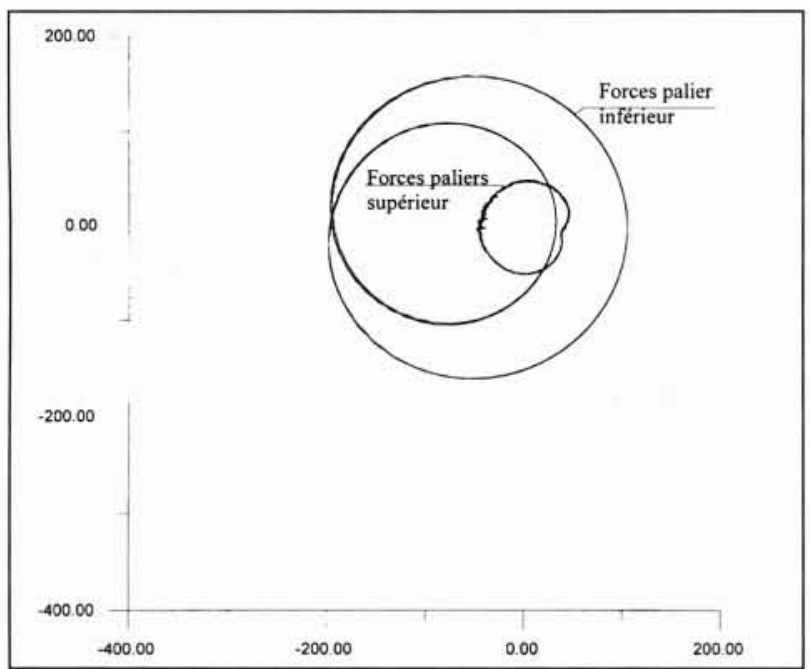

3. Réponse en force des paliers.

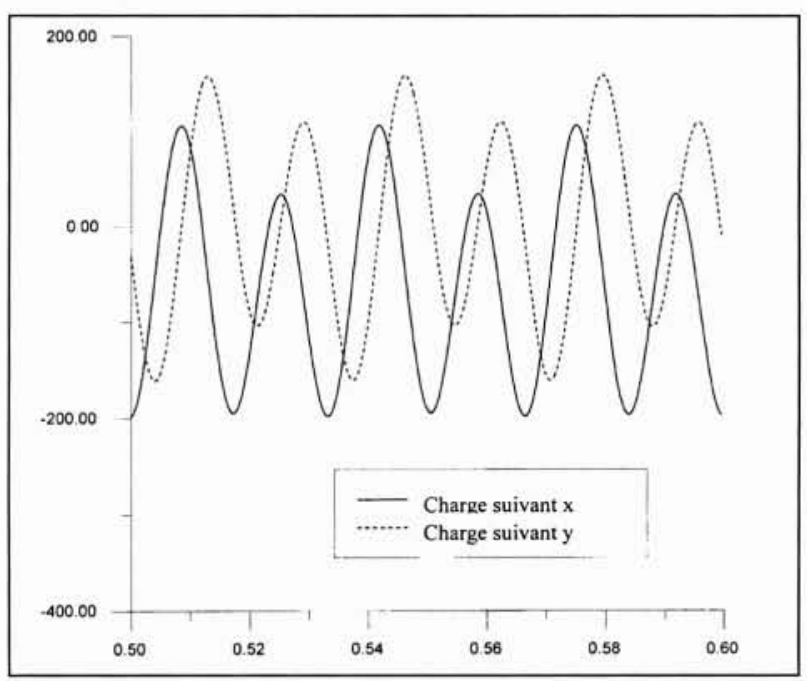

4. Charge fonction du temps palier inférieur.
L'influence de l'attraction magnétique est beaucoup plus apparente sur les charges apparaissant au niveau du palier supérieur. En effet la charge magnétique s'exerce à proximité de ce palier et le balourd est quant à lui géométriquement très éloigné. Cela se traduit par une force évoluant à la demi-fréquence (fig. 3 et 5 ) de l'arbre et peu influencée par le balourd.

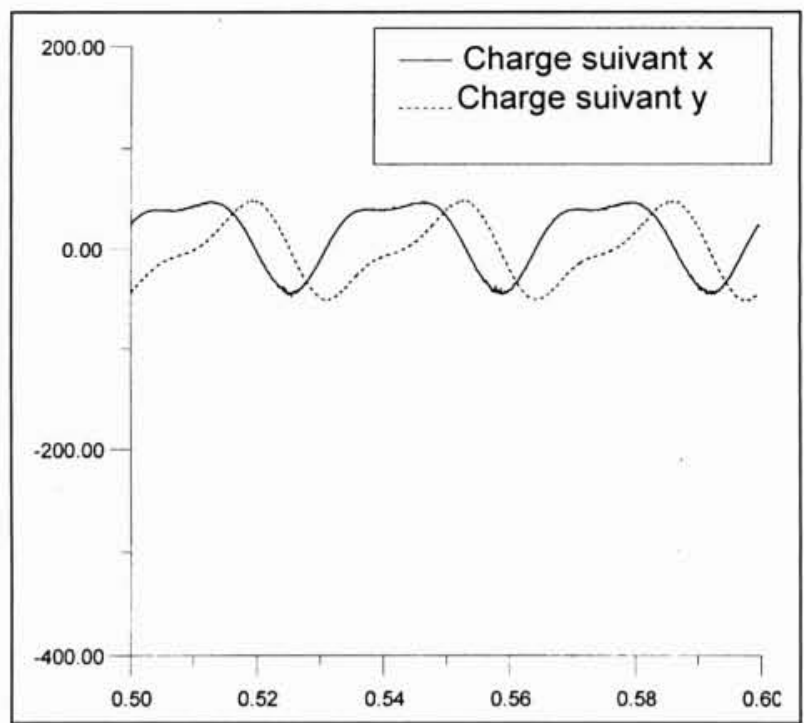

5. Charge fonction du temps palier supérieur.

\subsection{Trajectoires dans les paliers}

La figure 6 représente les trajectoires de l'arbre dans les deux paliers. Il s'agit de deux cercles ayant une excentricité proche de un, ce qui est caractéristique d'une instabilité de palier. Cela est bien entendu confirmé sur les figures 7 et 8 : l'arbre a un mouvement à la demi-fréquence ( 3 périodes de précession de l'arbre pour 6 rotations).

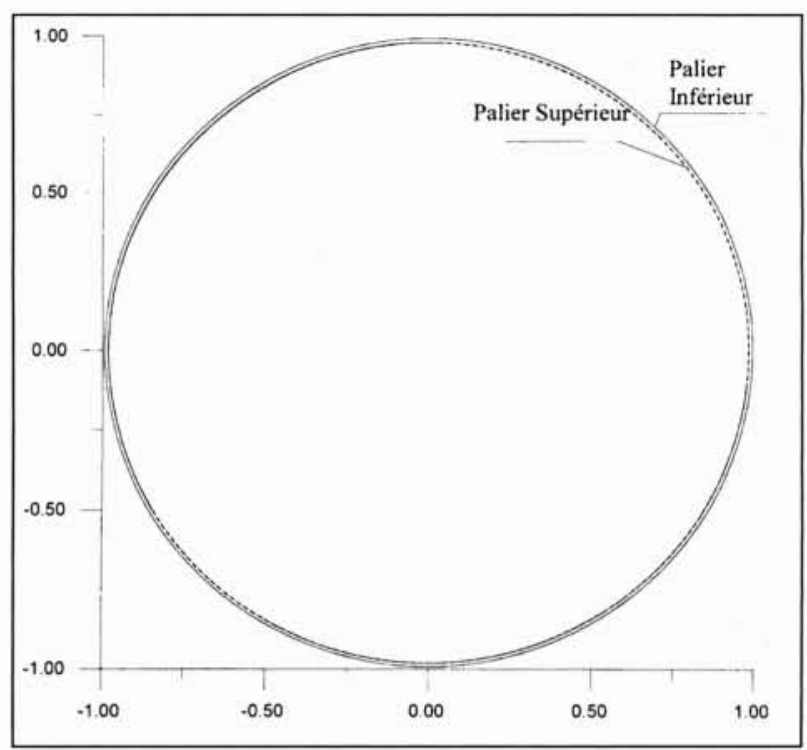

6. Orbites adimensionnées dans les paliers. 


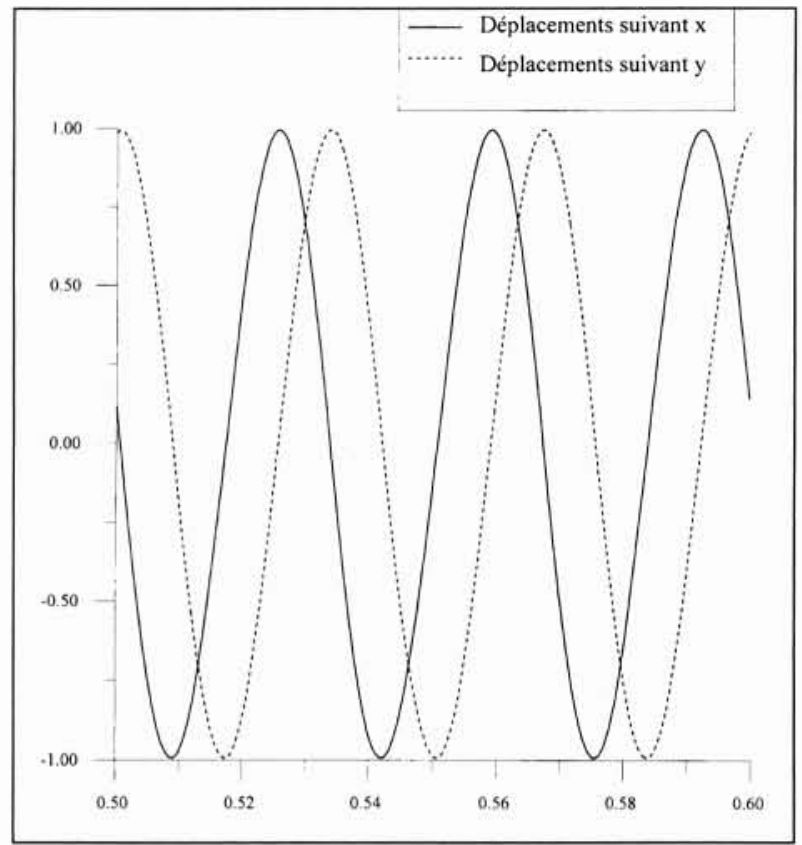

7. Amplitudes dans le palier inférieur.

\subsection{Balourd et Attraction magnétique}

Les figures 9 et 10 présentent respectivement la représentation cartésienne et l'évolution en fonction du temps des charges extérieures sur l'arbre (balourd et attraction magnétique).

Ces courbes confirment les remarques précédentes: les balourds agissent évidemment à la fréquence synchrone alors que les charges magnétiques suivent le mouvement de précession de l'arbre à demi fréquence et sont donc très déstabilisatrices.

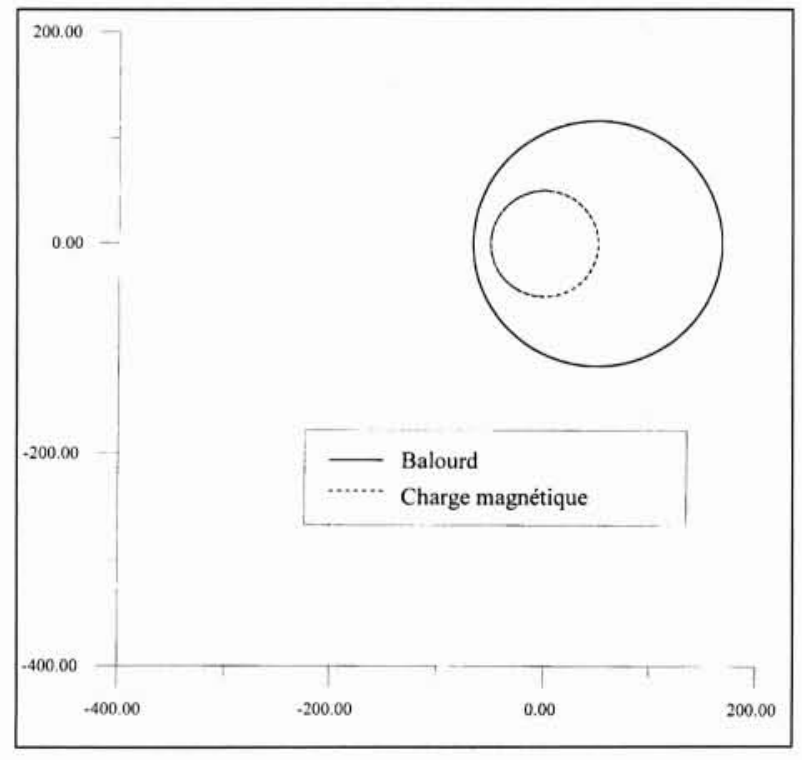

9. Balourd et charge magnétique

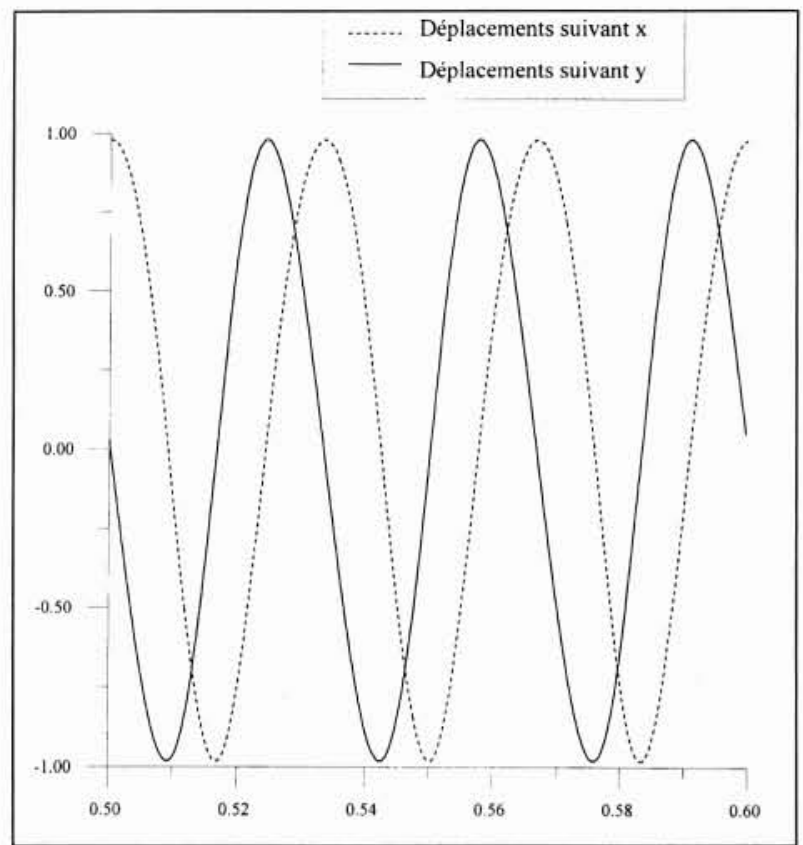

8. Amplitude dans le palier supérieur.

\section{IV —CONCLUSION}

Un modèle numérique d'arbre vertical monté dans deux paliers lubrifiés à l'eau a été développé. L'attraction magnétique et certains effets hydrauliques ont été pris en compte. Cette étude permet de confirmer le caractère instable de cet arbre monté dans deux paliers lisses. Cette instabilité est due d'une part à l'absence de fortes charges statiques radiales, et d'autre part à l'alimentation pressurisée des paliers $(4,5$ bars et 8 bars). En effet la rupture du film est un facteur stabili-

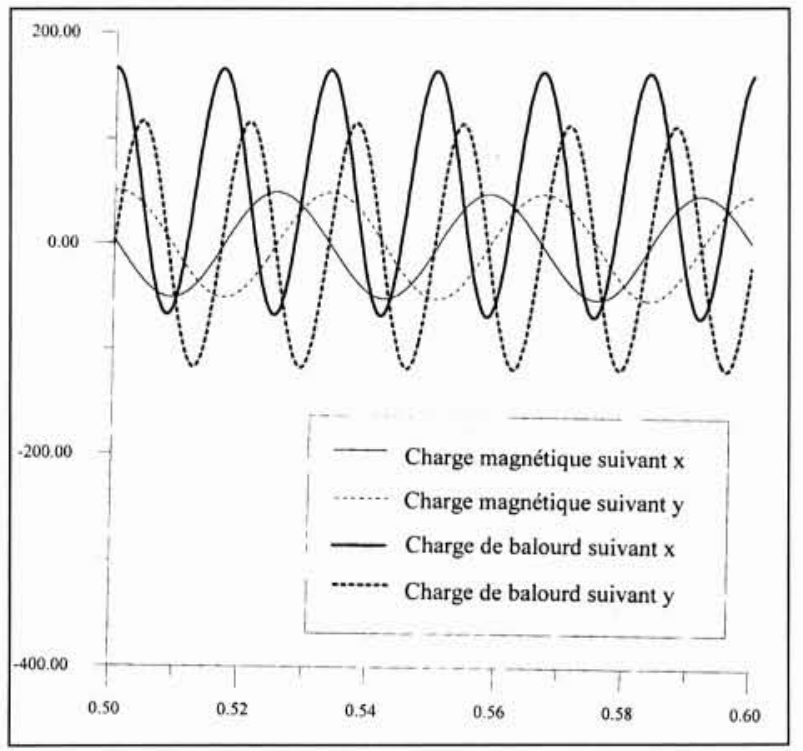

10. Balourd et charge magnétique fonction du temps 
sant, ces pressions d'alimentation, assez importantes, ont pour effet de diminuer (voire de supprimer) la zone de rupture du film.

Cette instabilité apparaît évidemment sur le mécanisme réel (turbopompe verticale) et peut poser quelquefois des problèmes de fiabilité en particulier en raison des rugosités réelles des paliers ou de conicité, due à l'usinage des paliers. Une étude, non présentée ici, a été effectuée avec des défauts de conicité dans les paliers. Ces défauts peuvent conduire à des amplitudes dynamiques très importantes.

Pour conclure, la.prédiction du comportement dynamique de cet arbre est très sensible. De petites perturbations sur certains paramètres peuvent conduire à de sérieux dommages.

\section{RÉFÉRENCES BIBLIOGRAPHIQUES}

[1] NewkIRK B.L., 1924. — "Shaft Whipping ». Gen. Electr. Rev., vol. 27, pp. 169-178.

[2] NewkiRK B.L., TAYLor H.D., 1925. - « Shaft whipping due to oil action in journal bearings ». Gen. Elect. Rev., p. 559.

[3] Hummel B.L., 1926. - « Krtitische Drehzahlen als folge der nachgiebigkeit des schmiermittels im lager». VDI-Forschift, p. 287.

[4] NEWKIRK B.L., 1931. - « Whiling balance shafts ». Third ICAM, Stockholm, Proc. 3, pp. 105-110.

[5] HoRI Y., 1959. - "A theory of oil whip ", Fith Japan Nat. Congress A. M. Proc. 395, 8(a) JAM 26, T81, pp. 189-198.

[6] Stodola A., 1925. - « Kritische wellenstörung infolge der nachgiebigkeit des oelpolsters im lager $»$. Scheizerische Bauzeitung, pp. 85-265.

[7] Holmes R., 1960. - « The vibration of a rigid shaft in short sleeve bearings». Jnal Mech. Eng. Sci., vol. 2, pp. 337-341.

[8] Sternlicht B., 1958. - «Elastic and damping properties of oil film journal bearing for application to unbalance vibration of calculation ". ASME, J. Applied Mech, vol. 80, p. 141.

[9] LUND J.W., 1965. - « The stability of an elastic rotor in journal bearings with flexible damped supports ». ASME, Jnal of Applied Mech., Dec. p. 911.

[10] Holmes R., 1963. — « Oil whirl characteristics of a rigid rotor in $360^{\circ}$ journal bearings ». Proc. IME 177, pp. 291-302.
[11] Holmes R., 1970. — « Non linear performance of turbine bearings ». Jnal of Mech. Eng. Sci., vol. n 6, p. 377.

[12] Lund J.W., SAIBel E., 1967. — « Oil whip whirl orbits of a rotor in sleeve bearings ». ASME, Jnal of Eng. for Ind., nov. p. 813 .

[13] ABDUL-WaHEd N., 1982. - «Comportement dynamique des paliers fluides. Etude linéaire et non linéaire ». Thèse de doctorat d'état, Université Claude Bernard, Lyon.

[14] Simon F., Frene J., 1991. — "Analysis for Incompressible Flow in Annular Pressure Seals ». ASME, Journal of tribology, vol. 114, pp. 431-438.

[15] Simon F., Frene J., 1990. — « Rotordynamic coefficients for turbulent annular misaligned seals " Third International Symposium on Transport Phenomena and Dynamics of Rotating Machinery, Hawaii, USA, pp. 289-304.

[16] Lucas V., Danaila S., Bonneau O., Frene J., 1994. «Roughness Influence on turbulent flow through annular seals ». Journal of Tribology ASME, vol. 116, April, pp. 321-327.

[17] Lucas V., Bonneau O., Frene J., 1996. - « Roughness Influence on the Turbulent Flow Through Annular Seals Including Inertia Effects ». Journal of Tribology ASME, vol. 118, January, pp. 175-182.

[18] Constantinescu V.N., Pan C.H.T., Smalley A.J., Vohr J.H., 1970. - « Lubrication phenomena in a film of low kinematic viscosity ». Rev. Roum. Sc. Techn. Mech. Appl., vol. $15, \mathrm{~N}^{\circ} 2$, pp. 479-502.

[19] Bonneau O., 1989. - « Comportement Statique et Dynamique de ligne d'Arbre Montée sur Paliers Fluides: Influence des Caractéristiques des Paliers ». Thèse de l'Université de Poitiers, soutenue le 2 février 1989.

[20] Bonneau O., Kassai A., Frene J., Der Hagopian J., 1989. - « Dynamical behavior of an elastic rotor with squeeze film damper ». Eurotrib Helsinki Finlande, juin, Proceeding vol. 4, pp. 145-149.

[21] Bonneau O., Frene J., 1994. - « Numerical study of a flexible rotor mounted in an active squeeze film damper $»$. Fourth International Conference On Rotor Dynamics, IFTOM Chicago, pp. 327-331.

[22] Pecheux B., Bonneau O., Frene J., 1997. - « Investigation about Electro Rheological Squeeze Film Damper Applied to Active Control of Rotor Dynamic ». International Journal of Rotating Machinery, Gordon \& Breach Science Publishers, Vol. 3, issue 1, January. 